\title{
Psychosocial and Aesthetic Advantages of Reconstruction after Prophylactic Mastectomy: A Quality of Life and Aesthetic Analysis
}

\author{
Michele A. Manahan, MD ${ }^{1}$ Pablo A. Baltodano, MD ${ }^{2}$ Myrna Eliann Reinhardt, BA, BM ${ }^{1}$ Li Xie, ScM ${ }^{3,4}$ \\ Justin C. Muste, $\mathrm{BA}^{5}$ Rehan Tajamal, MBBS ${ }^{1}$ Raja Mohan, $\mathrm{MD}^{1}$ José M. Flores, MPH \\ Carolyn Drogt, BS ${ }^{1}$ Carisa M. Cooney, $\mathrm{MPH}^{1}$ Gedge D. Rosson, MD ${ }^{1}$
}

\footnotetext{
${ }^{1}$ Department of Plastic and Reconstructive Surgery, Johns Hopkins University School of Medicine, Baltimore, Maryland

2 Division of Plastic Surgery, Albany Medical Center, Albany, New York

${ }^{3}$ Department of Biostatistics, Johns Hopkins Bloomberg School of Public Health, Baltimore, Maryland

${ }^{4}$ Department of Research Administration, Nemours Biomedical

Research, Wilmington, Delaware

${ }^{5}$ Division of Plastic Surgery, Albany Medical Center, Albany, New York

${ }^{6}$ Department of Epidemiology, Johns Hopkins Bloomberg School of Public Health, Baltimore, Maryland
}

J Reconstr Microsurg 2017;33:483-492.
Address for correspondence Gedge D. Rosson, MD, Department of Plastic and Reconstructive Surgery, Johns Hopkins University School of Medicine, McElderry 8161, 601 North Caroline Street, Baltimore, MD 21287 (e-mail: gedge@jhmi.edu).

\begin{abstract}
Keywords

- prophylactic mastectomy

- quality of life

- psychosocial wellbeing

Background Proper patient counseling regarding reconstruction after prophylactic mastectomy (PM) requires greater understanding of quality of life (QoL) and aesthetic outcomes. Our study evaluates these end points using the validated BREAST-Q and Garbay system.

Methods We performed a retrospective chart review of 1,254 mastectomy patients (July 2008 through July 2012). Of those with completed preoperative BREAST-Q and reconstruction with a minimum of 6-month follow-up, 18 underwent bilateral PM (BPM), 36 underwent contralateral PM (CPM), and 30 matched controls were selected who underwent unilateral therapeutic mastectomy (TM) with contralateral symmetry procedure. Preoperative and 6-month postreconstruction BREAST-Q scores were compared within and between groups. Subsequently, aesthetic evaluation of 21 of a group of randomly selected reconstructions (7 TM, 7 CPM, and 7 BPM) was performed by 14 plastic surgery researchers. Potential correlations between aesthetic evaluations and QoL outcomes were examined. Results Demographic characteristics, preoperative satisfaction scores, and postoperative morbidity rates were similar among all three groups. Although reconstruction after BPM was associated with improved aesthetic outcomes $(p<0.001)$, a significant postoperative increase in satisfaction with breasts was noted only in the TM group $(p=0.006)$. CPM was associated with improved psychosocial well-being $(p=0.039)$ in our study. No further significant differences were noted.

Conclusion Although BPM with reconstruction is associated with higher aesthetic outcomes compared with CPM or TM, QoL is not significantly increased. Reconstruction after CPM, but not BPM, is associated with increased psychosocial well-being. These valuable findings should be taken into account during preoperative counseling regarding elective PMs.
\end{abstract}

received

April 30, 2016

accepted after revision

March 23, 2017

published online

June 12, 2017
Copyright $\odot 2017$ by Thieme Medical Publishers, Inc., 333 Seventh Avenue, New York, NY 10001, USA. Tel: +1(212) 584-4662.
DOI https://doi.org/

$10.1055 / \mathrm{s}-0037-1602785$. ISSN 0743-684X. 
Breast cancer represents the most common nonskin malignancy and second leading cause of cancer-related deaths in American women. ${ }^{1-3}$ It is estimated that in the United States, during year 2013, a total of 232,340 women were diagnosed with breast cancer and 39,620 women died from this disease. ${ }^{1}$ Since breast cancer represents such a health care burden, several preventive and therapeutic strategies have been developed in an attempt to decrease the impact of this disease. In high-risk populations, prophylactic mastectomy (PM) reduces the risk of breast cancer 90 to $95 \% .{ }^{4,5}$ With such a significant reduction in the incidence of breast cancer and the increasing availability of genetic counseling, PM is being increasingly performed, and the number of patients undergoing breast reconstruction after PM is increasing concomitantly. ${ }^{6-8}$

There is also an increasing demand for patient-reported outcomes (PROs) in the field of breast reconstructive surgery. ${ }^{9,10}$ PRO data are critical to ensure adequate preoperative counseling and patient education by medical oncologists, genetic counselors, surgical oncologists, and plastic surgeons working with women considering PM followed by reconstruction. Although several studies have evaluated satisfaction and quality of life (QoL) of patients undergoing reconstruction after $\mathrm{PM},{ }^{11-31}$ these studies used nonvalidated questionnaires. ${ }^{32}$ To our knowledge, only one study has employed the validated BREAST- $\mathrm{Q}^{33}$ to evaluate satisfaction and QoL in the PM population. ${ }^{27}$ However, that study did not adjust for baseline QoL scores, making it difficult to elucidate whether the differences found in postoperative QoL scores are a result of the procedure or just a reflection of different preoperative QoL scores. Furthermore, the previous study did not include autologous reconstruction or correlations with the aesthetic quality of the reconstructions. $^{27}$

We analyzed the postoperative satisfaction and QoL outcomes of reconstruction after PM using the validated BREAST-Q instrument, adjusting for preoperative (baseline) QoL scores. In addition, we pursued a correlation between patient satisfaction and the aesthetic outcomes of reconstruction after PM. We hypothesize that patients undergoing reconstruction after bilateral PM (BPM), who typically do not require adjuvant therapies, will have heightened aesthetic results and, for that reason, higher levels of satisfaction than patients who undergo reconstruction after contralateral PM (CPM) or therapeutic mastectomies (TMs) with TM likely scoring the lowest because of challenges matching a native breast to a reconstruction.

\section{Patients and Methods}

\section{Patients}

\section{Inclusion Criteria}

Patients who underwent breast reconstruction between July 2008 and July 2012 at a single institution and completed the preoperative and 6-month postoperative BREAST-Q after final reconstruction were eligible for inclusion in the Institutional Review Board-approved protocol of this study. The 6-month postoperative BREAST-Q was completed 6 months or more after the definitive reconstruction (permanent implant or autologous tissue) for all patients. Three breast reconstruction populations were studied: patients who underwent BPM, CPM (one mastectomy for therapeutic local control and the contralateral mastectomy for prophylaxis), and a control population of patients who underwent TM (with contralateral surgery to optimize symmetry with the reconstructed breast). Symmetry procedures included augmentation, mastopexy, and/or reduction mammoplasty.

\section{Exclusion Criteria}

Patients who did not complete a preoperative BREAST-Q; did not undergo permanent reconstruction after BPM, CPM, or TM; or did not complete a 6-month postoperative BREAST-Q following reconstruction were excluded.

\section{Chart Review}

A retrospective chart review was performed to retrieve the following data: age at definitive reconstruction, body mass index (BMI), race, type of definitive reconstruction (implant or autologous), type of mastectomy (BPM, CPM, TM, either nipple sparing or nonnipple sparing), comorbidities, smoking status, prior chemotherapy, prior radiotherapy, reconstruction timing (immediate, staged, delayed), postoperative morbidity, revision surgeries, nipple reconstruction procedures, and postoperative digital photographs.

\section{Design}

Patients were consented and asked to complete the preoperative BREAST-Q questionnaire prior to undergoing mastectomy. Six months after their definitive breast reconstructive surgery, patients were asked to complete the postoperative BREAST-Q questionnaire. Patients were considered nonresponders if the responses could not be retrieved by 8 months postoperatively. Patient identification for inclusion as well as data collection and analysis were performed in a retrospective fashion.

Responses were scored according to the QScore Scoring Software (available at: https://webcore.mskcc.org/breastq/ scoreBQ.html) to generate a continuous variable ranging from 0 to 100 per domain. In this scoring system and for all scales, higher scores indicate greater satisfaction. ${ }^{34}$ Preoperative scores in each domain of the BREAST-Q were compared between the groups (BPM, CPM, and TM). Preoperative and postoperative scores were compared within the BPM, CPM, and TM groups. We also performed a subgroup analysis by reconstruction type (implant or autologous) for each one of the groups (BPM, CPM, and TM).

An exploratory analysis of the aesthetic outcomes of reconstruction after each type of mastectomy (BPM, CPM, and TM) was conducted. For this purpose, we used a random sequence generator (available at: http://www.random.org/ sequences/) to obtain a random sample of seven responders from each group (TM, CPM, and BPM). Then, we performed an aesthetic assessment of the 6-month postdefinitive reconstruction frontal and bilateral-oblique digital photographs. Fourteen blinded breast reconstruction researchers (excluding 
the operating surgeons) from the Johns Hopkins Avon Foundation Breast Center independently reviewed the photographs and scored aesthetic outcomes on a three-point modified Garbay scale (ranging from 0 to 2). ${ }^{35}$ The characteristics subscales comprise volume, shape, placement, inframammary fold, and scars. The scores were then averaged for tabulation and analysis.

\section{Statistical Analysis}

Baseline characteristics were compared between CPM, BPM, and TM recipients. Continuous variables were analyzed for normality. Normal data were compared using Student's t-test, while nonnormally distributed data were compared utilizing the Mann-Whitney $U$-test. Categorical variables were assessed using Pearson's chi-square or Fisher's exact tests. For the QoL assessment, between-group (BPM, CPM, and TM) scores on the BREAST-Q were compared using the one-way analysis of variance test. In addition, within-group scores (pre- vs. postoperative) were compared using the twosample independent Student's t-test.

For the aesthetic evaluation, the subscale scores ranging from 0 to 2 were averaged then compared using Student's $t$-test. In addition, the subscale averages were totaled to give an overall aesthetic score.

A $p<0.05$ criterion of statistical significance was employed for all tests. Statistical analysis was performed using $R$ Statistical Software (R Foundation for Statistical Computing) and Stata 12 (StataCorp. 2011. Stata Statistical Software: Release 12. StataCorp LP).

\section{Results}

\section{Populations and Response Rate}

During the study period, a total of 1,254 mastectomies were performed at the Johns Hopkins Avon Foundation Breast Center. Of these, a total of $93 \mathrm{PM}$ patients were eligible for the study. Three were diagnosed with contralateral breast cancer before the 6-month postdefinitive reconstruction time point and were excluded. The 6-month postoperative BREAST-Q questionnaire was offered to 90 PM patients, of whom 54 patients successfully completed the questionnaire (18 BPM patients; 36 CPM patients), for an overall response rate of $60 \%$. We identified 30 controls undergoing TM and contralateral symmetry procedure who had completed both the pre- and 6-month postoperative BREAST-Q questionnaires, matched on age, BMI, type of reconstruction, and operative date. A total of 84 patients were finally included in this study (18 BPM, 36 CPM, 30 TM).

\section{Patient Demographics}

Baseline demographic variables were similar between the BPM, CPM, and TM groups (- Table 1). As expected, none of the BPM patients received radiation therapy, and the number of irradiated patients was not significantly different between TM and CPM groups $(p=0.170)$. Importantly, the rates of comorbidities, type of reconstruction, reconstruction timing, postoperative morbidity, revision and nipple reconstruction surgery, nipple-sparing mastectomy, and nipple reconstruc- tion procedures were similar between all groups. The mean preoperative BREAST-Q scores were similar among the three groups (BPM, CPM, and TM) across all the domains of the BREAST-Q (-Table $\mathbf{1}$ ).

\section{BREAST-Q Scores}

\section{Bilateral Prophylactic Mastectomy Group}

Within this group $(n=18)$, we compared the pre- to postoperative mean scores and found that the levels of satisfaction/QoL did not change significantly for any of the domains analyzed: satisfaction with breasts, psychosocial well-being, chest well-being, and sexual well-being (-Table 2 ).

\section{Contralateral Prophylactic Mastectomy Group}

Within this group ( $n=36$ ), we compared the pre- to postoperative mean scores and found a statistically significant postoperative improvement in the psychosocial well-being $(p=0.039)$ domain. No significant change was noted in the satisfaction with breasts, chest well-being, or sexual wellbeing domains ( - Table 2 ).

\section{Therapeutic Mastectomy Group (Controls)}

Within this group ( $n=37$ ), we compared pre- to postoperative mean scores and found a statistically significant postoperative improvement in the satisfaction with breasts (0.006) domain. No significant changes were noted in the other domains (- Table 2).

\section{Subgroup Analyses}

\section{Autologous Breast Reconstruction}

We compared pre- to postoperative mean scores for each BREAST-Q domain in the population undergoing autologous breast reconstruction after BPM, CPM, and TM. This subgroup analysis demonstrated a statistically significant improvement in the psychosocial well-being domain for the BPM group ( $p=0.044$ ) and the satisfaction with breast domain for the TM group $(p=0.010)$. There were no other significant findings in any of the other domains for the BPM, CPM, or TM groups (-Table 3 ).

\section{Implant-Based Breast Reconstruction}

Subsequently, we compared pre- to postoperative mean scores for each BREAST-Q domain in the population undergoing implant-based breast reconstruction after BPM, CPM, and TM. This subgroup analysis did not demonstrate significant differences in any of the domains analyzed (-Table 4).

\section{Aesthetic Outcomes}

Aesthetic outcomes were evaluated for each of the 21 randomly selected patients ( 7 from each group) and then compared between the groups. When the overall aesthetic scores were compared between the groups (- Table 5), BPM showed significantly better overall aesthetic outcomes compared with reconstruction after TM $(p<0.001)$. However, CPM did not show a significant overall aesthetic difference 
Table 1 Population characteristics

\begin{tabular}{|c|c|c|c|c|}
\hline Variable & $\mathrm{BPM}(n=18)$ & CPM $(n=36)$ & $\mathrm{TM}(n=30)$ & $p$-Value \\
\hline \multicolumn{5}{|c|}{ Age at final reconstruction, $y$} \\
\hline Mean & 47.2 & 50.9 & 53.2 & \multirow[t]{2}{*}{0.057} \\
\hline Range & $38-56$ & $43-59$ & $44-62$ & \\
\hline \multicolumn{5}{|l|}{ BMI, $\mathrm{kg} / \mathrm{m}^{2}$} \\
\hline Mean & 27.3 & 26.2 & 25.3 & \multirow[t]{2}{*}{0.438} \\
\hline Range & $20.8-33.8$ & $21.0-31.4$ & $20.5-30.1$ & \\
\hline \multicolumn{5}{|l|}{ Race } \\
\hline White & $17(94.4 \%)$ & $33(91.7 \%)$ & $21(70.0 \%)$ & \multirow[t]{4}{*}{0.120} \\
\hline Black & $1(5.6 \%)$ & $3(8.3 \%)$ & $5(16.7 \%)$ & \\
\hline Asian & 0 & 0 & $1(3.3 \%)$ & \\
\hline Other & 0 & 0 & $3(10.0 \%)$ & \\
\hline \multicolumn{5}{|l|}{ Diabetes } \\
\hline Yes & 0 & $2(5.6 \%)$ & $2(6.7 \%)$ & 0.678 \\
\hline \multicolumn{5}{|l|}{ Hypertension } \\
\hline Yes & $1(5.6 \%)$ & $5(13.9 \%)$ & $5(16.7 \%)$ & 0.642 \\
\hline \multicolumn{5}{|l|}{ Thyroid disease } \\
\hline Yes & $4(22.2 \%)$ & $6(16.7 \%)$ & $4(13.3 \%)$ & 0.647 \\
\hline \multicolumn{5}{|l|}{ Smoker } \\
\hline Yes & 0 & $4(11.1 \%)$ & $1(3.3 \%)$ & 0.275 \\
\hline \multicolumn{5}{|c|}{ Type of reconstruction } \\
\hline Autologous & $10(55.6 \%)$ & $22(61.1 \%)$ & $22(73.3 \%)$ & \multirow[t]{2}{*}{0.401} \\
\hline Implant & $8(44.4 \%)$ & $14(38.9 \%)$ & $8(26.7 \%)$ & \\
\hline \multicolumn{5}{|l|}{ Prior chemotherapy } \\
\hline Yes & 0 & $18(50.0 \%)$ & $11(36.7 \%)$ & $0.277^{\mathrm{a}}$ \\
\hline \multicolumn{5}{|l|}{ Prior radiotherapy } \\
\hline Yes & 0 & $12(33.3 \%)$ & $15(50.0 \%)$ & $0.170^{\mathrm{a}}$ \\
\hline \multicolumn{5}{|l|}{ Reconstruction timing } \\
\hline Immediate & $4(22.2 \%)$ & $6(16.7 \%)$ & $8(26.7 \%)$ & \multirow[t]{3}{*}{0.117} \\
\hline Staged & $14(77.8 \%)$ & $29(80.6 \%)$ & $17(56.7 \%)$ & \\
\hline Delayed & 0 & $1(2.8 \%)$ & $5(16.7 \%)$ & \\
\hline \multicolumn{5}{|c|}{ Any postoperative morbidity } \\
\hline Yes & $5(27.8 \%)$ & $14(38.9 \%)$ & $15(50.0 \%)$ & 0.318 \\
\hline \multicolumn{5}{|l|}{ Revision surgery } \\
\hline Yes & $5(27.8 \%)$ & $19(52.8 \%)$ & $14(46.7 \%)$ & 0.216 \\
\hline \multicolumn{5}{|c|}{ Nipple-sparing mastectomy } \\
\hline Yes & $6(33.3 \%)$ & $10(27.8 \%)$ & 7 (23.3\%) & 0.752 \\
\hline \multicolumn{5}{|l|}{ Nipple reconstruction } \\
\hline Yes & 0 & $6(16.7 \%)$ & $5(16.7 \%)$ & 0.210 \\
\hline \multicolumn{5}{|c|}{ Preoperative satisfaction with breasts } \\
\hline Mean score \pm SD & $49.8 \pm 22.9$ & $53.7 \pm 25.9$ & $59.1 \pm 18.5$ & 0.183 \\
\hline \multicolumn{5}{|c|}{ Preoperative psychosocial well-being } \\
\hline Mean score \pm SD & $64.5 \pm 14.4$ & $66.1 \pm 18.2$ & $70.3 \pm 19.3$ & 0.416 \\
\hline
\end{tabular}


Table 1 (Continued)

\begin{tabular}{|l|l|l|l|l|}
\hline Variable & BPM $(\boldsymbol{n}=\mathbf{1 8})$ & CPM $(\boldsymbol{n}=\mathbf{3 6})$ & TM $(\boldsymbol{n}=\mathbf{3 0})$ & $p$-Value \\
\hline Mean score \pm SD & $82.3 \pm 11.9$ & $77.6 \pm 15.2$ & $77.5 \pm 14.6$ & 0.524 \\
\hline Preoperative sexual well-being & \multicolumn{1}{l}{0.092} \\
\hline Mean score \pm SD & $51.2 \pm 13.1$ & $53.7 \pm 18.1$ & $54.9 \pm 21.9$ & 0.9 \\
\hline
\end{tabular}

Abbreviations: BPM, bilateral prophylactic mastectomy; CPM, contralateral prophylactic mastectomy; SD, standard deviation; TM, therapeutic mastectomy.

${ }^{\mathrm{a} C o m p a r i s o n}$ between the TM and CPM groups only.

from TM $(p=0.194)$. In addition, both CPM and BPM demonstrated better volumetric aesthetics than TM $(p<0.01$, for both). The scores for shape and placement were significantly higher in the BPM group ( $p<0.01$ and $p<0.05$, respectively) but not in the CPM group when compared with TM. The scars subscale scores did not differ significantly between any of the groups. Interestingly, reconstructions after CPM $(p<0.001)$, but not after BPM $(p=0.508)$, were scored significantly lower in regard to the inframammary fold when compared with TM. Of note, these overall aesthetic associations prevailed even after sensitivity analysis excluding the previously irradiated patients.

\section{Discussion}

The decision for PM is certainly challenging for patients considered at high risk for breast cancer and for those who may consider the procedure even without a large yield in risk reduction. Several factors play a role in the decision-making process. These include potential survival benefits, reconstructive options and outcomes, QoL, and physician counseling. ${ }^{13,26}$

Regarding risk reduction, Lostumbo et al's Cochrane systematic review found no randomized controlled trials regarding PM. Existing studies of other designs demonstrated BPM reduced incidence and mortality of breast cancer in high-risk patients, while CPM reduced incidence but not mortality. ${ }^{36}$ It would seem then that the decision for BPM would hinge more critically upon risk reduction, while the decision for CPM would require more careful consideration of the other aspects of the procedure such as reconstructive options and outcomes and patient-reported QoL associated with the proposed procedures. This would mean patients receiving BPM really experience less of a choice in

Table 2 Preoperative to postoperative BREAST-Q scores comparison within the bilateral, contralateral, and therapeutic mastectomy groups

\begin{tabular}{|c|c|c|c|}
\hline BREAST-Q domains & $\begin{array}{l}\text { Preoperative } \\
\text { (mean score } \pm \text { SD) }\end{array}$ & $\begin{array}{l}\text { Postoperative } \\
\text { (mean score } \pm \text { SD) }\end{array}$ & $p$-Value \\
\hline \multicolumn{4}{|l|}{ BPM $(n=18)$} \\
\hline Satisfaction with breasts & $49.8 \pm 22.9$ & $61.3 \pm 19.2$ & 0.112 \\
\hline Psychosocial well-being & $64.5 \pm 14.4$ & $72.3 \pm 23.3$ & 0.233 \\
\hline Physical well-being: chest & $82.3 \pm 11.9$ & $80.4 \pm 14.2$ & 0.668 \\
\hline Sexual well-being & $51.2 \pm 13.1$ & $53.8 \pm 27.0$ & 0.728 \\
\hline \multicolumn{4}{|l|}{ CPM $(n=36)$} \\
\hline Satisfaction with breasts & $53.7 \pm 25.9$ & $62.8 \pm 21.7$ & 0.108 \\
\hline Psychosocial well-being & $66.1 \pm 18.2$ & $76.8 \pm 24.4$ & $0.039^{\mathrm{a}}$ \\
\hline Physical well-being: chest & $77.6 \pm 15.2$ & $78.0 \pm 17.2$ & 0.914 \\
\hline Sexual well-being & $53.7 \pm 18.1$ & $55.4 \pm 28.3$ & 0.765 \\
\hline \multicolumn{4}{|l|}{$\operatorname{TM}(n=30)$} \\
\hline Satisfaction with breasts & $59.1 \pm 18.5$ & $73.4 \pm 20.6$ & $0.006^{\mathrm{b}}$ \\
\hline Psychosocial well-being & $70.3 \pm 19.3$ & $77.1 \pm 20.6$ & 0.195 \\
\hline Physical well-being: chest & $77.5 \pm 14.6$ & $71.2 \pm 18.4$ & 0.145 \\
\hline Sexual well-being & $54.9 \pm 21.9$ & $60.2 \pm 23.8$ & 0.381 \\
\hline
\end{tabular}

Abbreviations: BPM, bilateral prophylactic mastectomy; CPM, contralateral prophylactic mastectomy; SD, standard deviation; TM, therapeutic mastectomy.

Note: Score ranging from 0 to 100 .

${ }^{\mathrm{a}} \mathrm{p}<0.05$.

${ }^{\mathrm{b}} p<0.01$. 
Table 3 Preoperative and postoperative BREAST-Q scores comparison within the bilateral, contralateral, and therapeutic mastectomy groups among patients undergoing autologous breast reconstructions

\begin{tabular}{|l|l|l|l|}
\hline BREAST-Q domains & $\begin{array}{l}\text { Preoperative } \\
\text { (mean score } \pm \text { SD) }\end{array}$ & $\begin{array}{l}\text { Postoperative } \\
\text { (mean score } \pm \text { SD) }\end{array}$ \\
\hline BPM $(n=10)$ & $64.6 \pm 21.0$ & 0.070 \\
\hline Satisfaction with breasts & $44.0 \pm 26.5$ & $78.2 \pm 22.2$ & $0.044^{\text {a }}$ \\
\hline Psychosocial well-being & $60.3 \pm 13.8$ & $82.7 \pm 15.3$ & 0.665 \\
\hline Physical well-being: chest & $79.8 \pm 14.2$ & $66.5 \pm 24.6$ & 0.089 \\
\hline Sexual well-being & $48.9 \pm 16.6$ & & 0.434 \\
\hline CPM ( $n=22)$ & $64.4 \pm 16.8$ & 0.104 \\
\hline Satisfaction with breasts & $59.5 \pm 23.9$ & $78.3 \pm 22.1$ & 0.643 \\
\hline Psychosocial well-being & $68.3 \pm 17.5$ & $78.7 \pm 16.1$ & 0.569 \\
\hline Physical well-being: chest & $77.6 \pm 14.2$ & $56.6 \pm 25.4$ & \\
\hline Sexual well-being & $60.2 \pm 14.5$ & & $0.010^{\mathrm{b}}$ \\
\hline TM ( $n=22)$ & $75.5 \pm 20.3$ & 0.197 \\
\hline Satisfaction with breasts & $59.7 \pm 18.7$ & $78.7 \pm 20.1$ & 0.441 \\
\hline Psychosocial well-being & $70.8 \pm 20.2$ & $75.2 \pm 16.4$ & 0.486 \\
\hline Physical well-being: chest & $78.8 \pm 14.1$ & $59.6 \pm 23.8$ & \\
\hline Sexual well-being & $54.7 \pm 22.5$ & & \\
\hline
\end{tabular}

Abbreviations: BPM, bilateral prophylactic mastectomy; CPM, contralateral prophylactic mastectomy; SD, standard deviation; TM, therapeutic mastectomy.

Note: Score ranging from 0 to 100 .

${ }^{\mathrm{a}} \mathrm{p}<0.05$

${ }^{\mathrm{b}} \mathrm{p}<0.01$.

Table 4 Preoperative and postoperative BREAST-Q scores comparison within the bilateral, contralateral, and therapeutic mastectomy groups among patients undergoing implant-based breast reconstructions

\begin{tabular}{|l|l|l|l|}
\hline BREAST-Q domains & $\begin{array}{l}\text { Preoperative } \\
\text { (mean score } \pm \text { SD) }\end{array}$ & $\begin{array}{l}\text { Postoperative } \\
\text { (mean score } \pm \text { SD) }\end{array}$ & -Value \\
\hline BPM $(n=8)$ & $57.1 \pm 17.1$ & 0.988 \\
\hline Satisfaction with breasts & $57.0 \pm 16.1$ & $65.0 \pm 23.9$ & 0.637 \\
\hline Psychosocial well-being & $69.7 \pm 14.2$ & $77.6 \pm 13.1$ & 0.171 \\
\hline Physical well-being: chest & $85.5 \pm 8.1$ & $37.8 \pm 21.7$ & 0.069 \\
\hline Sexual well-being & $53.8 \pm 7.8$ & $60.4 \pm 28.2$ & 0.142 \\
\hline CPM ( $n=14)$ & $74.4 \pm 28.4$ & 0.210 \\
\hline Satisfaction with breasts & $44.5 \pm 27.2$ & $76.9 \pm 19.3$ & 0.743 \\
\hline Psychosocial well-being & $62.6 \pm 19.3$ & $53.3 \pm 33.5$ & 0.342 \\
\hline Physical well-being: chest & $79.2 \pm 17.2$ & & \\
\hline Sexual well-being & $43.0 \pm 18.6$ & $67.6 \pm 21.4$ & 0.340 \\
\hline TM (n=8) & $72.4 \pm 22.4$ & 0.736 \\
\hline Satisfaction with breasts & $57.6 \pm 19.0$ & $60.0 \pm 20.2$ & 0.150 \\
\hline Psychosocial well-being & $68.8 \pm 18.0$ & $61.5 \pm 25.2$ & 0.616 \\
\hline Physical well-being: chest & $73.8 \pm 16.0$ & $55.5 \pm 21.4$ & \\
\hline Sexual well-being & & & \\
\hline
\end{tabular}

Abbreviations: BPM, bilateral prophylactic mastectomy; CPM, contralateral prophylactic mastectomy; SD, standard deviation; TM, therapeutic mastectomy.

Note: Score ranging from 0 to 100 . 

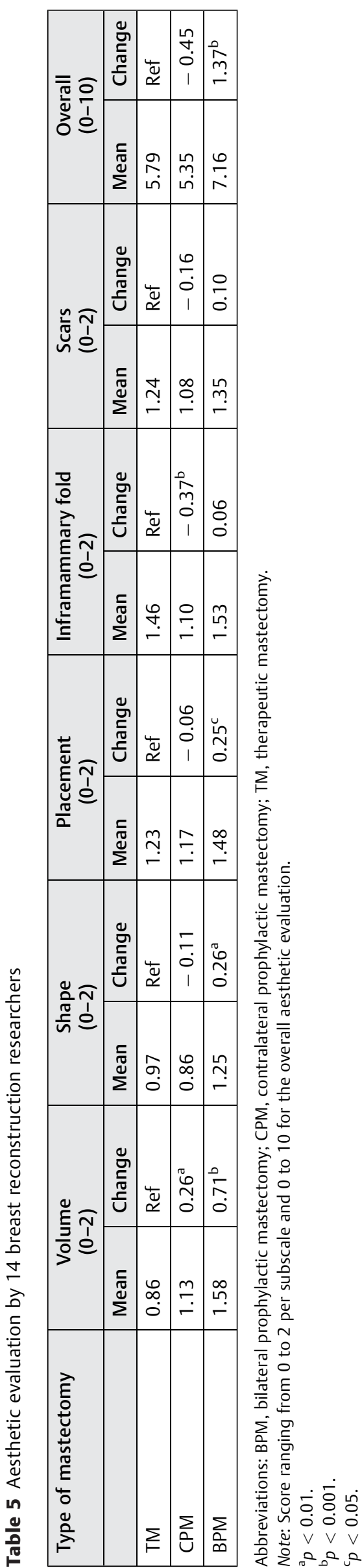

the matter since, such as a TM, they are undergoing a procedure which could be lifesaving. CPM patients may feel more in control of the decision to proceed with prophylactic surgery. In this light, several of our findings are relevant.

Potentially, the most important of our findings are that CPM, regardless of reconstruction type, and BPM with autologous reconstruction are associated with significant increases in psychosocial well-being (-Tables 2 and 3), and PM does not decrease QoL scores. In addition, our aesthetic assessment revealed improved aesthetic outcomes for BPM patients. From this, we can both reassure the BPM patient feeling somewhat cornered by the need for removal of both breasts while still healthy, and aid the CPM patient in making a weighty decision, which may not impact overall survival.

One of the advantages of our study, fairly unique in the existing literature to date, is the inclusion of both pre- and postoperative Breast-Q scores. The baseline (preoperative) comparison revealed that the psychosocial well-being associated with BPM, CPM, and TM was similar. This means that patients at high risk for breast cancer (BPM) basically have a baseline QoL impairment similar to that noted in patients diagnosed with breast cancer (CPM and TM). This is remarkable as it implies that the concern for being high risk for breast cancer produces a psychosocial impairment of similar magnitude to the diagnosis of breast cancer. All the other baseline QoL domains were also similar among the groups, which demonstrate that the groups started at comparable QoL levels and allow optimal assessment of their postoperative QoL outcomes.

Looking more closely at our cadre of findings in the BPM group, a group we have shown to be facing a similar QoL burden to actual cancer patients preoperatively, we found that performance of BPM is not associated with significant changes in QoL (-Table 2). To put this finding into context, one should also consider the results of the subgroup and aesthetic analyses, along with the previously mentioned survival benefits described in the literature. Taking these analyses into account, we could conclude that BPM represents an option that reduces the incidence and mortality of breast cancer (based on the existing literature), while at the same time increasing the psychosocial well-being of patients reconstructed with autologous tissue ( - Table 3 ). In addition, reconstruction after BPM was considered an option that renders heightened aesthetic outcomes when judged by third-party evaluators ( - Table 5). Similar aesthetic findings have been reported in the literature. ${ }^{11,37}$ This might reassure currently healthy patients reluctant to embark upon surgery with a potential for significant impact on body image.

Our findings related to CPM also need to be put into context. Based on the existing literature, although CPM does not consistently reduce the mortality secondary to breast cancer, it does reduces the incidence of contralateral breast cancer. ${ }^{36}$ The enhanced psychosocial well-being we found associated with undergoing CPM suggests that decreasing risk of repeat cancer diagnosis is important to patients despite absence of a survival benefit. On the contrary, it was interesting to find that CPM demonstrates similar 
aesthetic outcomes to unilateral TM with contralateral symmetry procedure. This is a particularly important finding since many patients express a desire for CPM to optimize postoperative aesthetic results, especially symmetry. Our findings imply that CPM represents an option that not only reduces the incidence of contralateral breast cancer but also improves the psychosocial well-being of patients with unilateral cancer. Both of these advantages are provided by CPM without significantly changing the aesthetics or the patients' satisfaction with their breasts. However, based on our findings, patients may need to be cautioned not to choose CPM solely for aesthetic reasons.

With regard to our finding that TM is associated with increased satisfaction with breasts, one might postulate that the increase in this domain of the TM group may be related to patient expectations. Pusic et al have previously reported that patient expectations play a fundamental role and have a significant impact on QoL and patient satisfaction after breast reconstruction. ${ }^{38}$ Patients expect improvement with plastic surgery, even if it is reconstruction; therefore, this might explain why patients in the TM group demonstrated BREAST-Q improvements postoperatively. The psychological effect of cancer removal might also play a role in patients' satisfaction and QoL (i.e., patients might be more satisfied with cancer-free reconstructed breasts than with diseased native breasts). Along these lines, recent literature has reported that cancer diagnosis by itself can have a negative impact on QOL. ${ }^{39,40}$ Hence, intuitively, we can expect cancer removal to help revert the detrimental effects associated with the diagnosis, with this being reflected as increased satisfaction with breasts. This may explain the differences between the TM and BPM groups.

The presence of cancer may lead to different consequences than risk of cancer. More aggressive mastectomies (skin flap thickness, margins of dissection) may be noted after therapeutic rather than PMs. This is supported by findings that BPM overall aesthetics are rated superior to either TM or CPM group. Similarly, inframammary fold aesthetics are rated superior in BPM compared with CPM. However, we expect some differences in QOL scores between TM and CPM groups may be attributed to a bias in which patients self-select into TM and CPM groups. There is likely a different approach to breasts and breast disease that leads patients to choose TM or CPM. This may account for the differences seen in our study between TM and CPM groups when examined from the perspective of "cancer treatment."

In summary, our study carries several advantages and sheds light on the psychosocial and aesthetic benefits of reconstruction after PM. Although there are several published studies that evaluate QOL in PM patients, ${ }^{11-31}$ to our knowledge, our study is the first BREAST-Q-based analysis assessing the BPM, CPM, and TM populations while taking the preoperative (baseline) QoL scores into account. Research has shown that preoperative BREAST-Q scores might vary significantly among different groups of patients seeking breast reconstruction, ${ }^{41}$ for that reason assessing both the preoperative scores and the postoperative scores becomes critical when comparing postoperative scores. Furthermore, it is also the first BREAST-Q study including a stratified analysis to determine the impact of the reconstruction type on the QoL outcomes of reconstruction after PM. This is especially true for the autologous breast reconstruction group, which has not been analyzed by previous BREAST-Q studies assessing QoL after PM. ${ }^{27}$ Further, it is interesting to note that the QoL differences identified in our study are clinically significant, as they are greater than the expected minimally important clinical difference. Norman et al concluded in a systematic review of QoL outcomes articles that the minimal important difference is one-half of a standard deviation, which in our study was approximately 10 BREAST$Q$ points. ${ }^{42}$ This was true for all the significant BREAST-Q associations identified in our study.

Finally, our study is the first BREAST-Q-based study establishing an exploratory correlation between satisfaction/QoL scores and aesthetic outcomes of reconstruction after PM. A final but nonetheless important advantage is that the patients in our study were evaluated at the same time point (6-month postdefinitive reconstruction), which allowed the groups to have similar rates of revision surgeries and nipple reconstruction procedures, enhancing their comparability.

Although our study carries the aforementioned advantages, it also carries some limitations, including those inherent to any retrospective analysis. Among those limitations, we need to mention that our study contains data from a single institution (the Johns Hopkins Avon Foundation Breast Center), where standard protocols and techniques for mastectomy and reconstruction may differ from other institutions. Another limitation of our study is the relatively small sample size, which provides limited power to detect additional interesting associations. We believe that further prospective studies using the validated BREAST-Q instrument should be conducted to validate our findings and further explore potential associations with a larger sample size. Along these lines, we believe that BPM might be associated with a statistically significant increase in satisfaction with breasts if a larger sample size is analyzed. We would also like to further explore the differences we began to find when our study populations were subdivided by reconstruction type. These and some other interesting associations might be evident with a larger sample size.

Clinical relevance was the determinant of our subgroups. Although fewer divisions and larger groups might predispose toward more statistically significant numbers, this might have obscured the clinical relevance by grouping dissimilar patients together. We feel our study reveals valuable information and may serve as a guide for future studies in this important area. Longer follow-up or subgroup analysis by other factors such as age or BMI may also facilitate understanding of these issues.

We are intrigued by our aesthetic outcomes and would like to more fully explore these issues as well. This analysis would benefit from inclusion of preoperative photographs as well as assessment of the potential impact of nipple-sparing techniques, reconstruction type, adjuvant radiation therapy, and other technical variations. 


\section{Conclusion}

CPM, regardless of reconstruction type, and BPM with autologous reconstruction are associated with significant increases in psychosocial well-being, and PM does not decrease QoL scores. In addition, our aesthetic assessment revealed improved aesthetic outcomes for BPM patients versus CPM and TM groups. From this, we can both reassure the BPM patient feeling concerned by the need for removal of both breasts while still healthy, and aid the CPM patient in making a weighty decision, which may not impact overall survival. These valuable findings should be taken into account by surgeons seeking to deliver optimal preoperative counseling.

\section{Acknowledgments}

The coauthors acknowledge the following institutional support: Department of Plastic Surgery, Johns Hopkins University receives research support from LifeCell Corp. (Branchburg, NJ) and educational support from LifeCell Corp. (Branchburg, NJ), Sientra Corp. (Santa Barbara, CA), and TEI Biosciences (Boston, MA), which make products that are used in plastic surgery reconstruction procedures.

\section{References}

1 Siegel R, Naishadham D, Jemal A. Cancer statistics, 2013. CA Cancer J Clin 2013;63(01):11-30

2 Greenlee RT, Murray T, Bolden S, Wingo PA. Cancer statistics, 2000. CA Cancer J Clin 2000;50(01):7-33

3 Centers for Disease Control and Prevention (CDC). Decline in breast cancer incidence-United States, 1999-2003. MMWR Morb Mortal Wkly Rep 2007;56(22):549-553

4 Hartmann LC, Schaid DJ, Woods JE, et al. Efficacy of bilateral prophylactic mastectomy in women with a family history of breast cancer. N Engl J Med 1999;340(02):77-84

5 Salhab M, Bismohun S, Mokbel K. Risk-reducing strategies for women carrying BRCA1/2 mutations with a focus on prophylactic surgery. BMC Womens Health 2010;10:28

6 Han E, Johnson N, Glissmeyer M, et al. Increasing incidence of bilateral mastectomies: the patient perspective. Am J Surg 2011; 201(05):615-618

7 Tuttle TM, Abbott A, Arrington A, Rueth N. The increasing use of prophylactic mastectomy in the prevention of breast cancer. Curr Oncol Rep 2010;12(01):16-21

8 Tuttle TM, Habermann EB, Grund EH, Morris TJ, Virnig BA. Increasing use of contralateral prophylactic mastectomy for breast cancer patients: a trend toward more aggressive surgical treatment. J Clin Oncol 2007;25(33):5203-5209

9 Cano SJ, Browne JP, Lamping DL. Patient-based measures of outcome in plastic surgery: current approaches and future directions. Br J Plast Surg 2004;57(01):1-11

10 Dean C, Chetty U, Forrest AP. Effects of immediate breast reconstruction on psychosocial morbidity after mastectomy. Lancet 1983;1(8322):459-462

11 de la Peña-Salcedo JA, Soto-Miranda MA, Lopez-Salguero JF. Prophylactic mastectomy: is it worth it? Aesthetic Plast Surg 2012;36(01):140-148

12 Geiger AM, West CN, Nekhlyudov L, et al. Contentment with quality of life among breast cancer survivors with and without contralateral prophylactic mastectomy. J Clin Oncol 2006;24(09): $1350-1356$
13 Grann VR, Panageas KS, Whang W, Antman KH, Neugut AI. Decision analysis of prophylactic mastectomy and oophorectomy in BRCA1-positive or BRCA2-positive patients. J Clin Oncol 1998; 16(03):979-985

14 Brandberg Y, Arver B, Lindblom A, Sandelin K, Wickman M, Hall P. Preoperative psychological reactions and quality of life among women with an increased risk of breast cancer who are considering a prophylactic mastectomy. Eur J Cancer 2004;40(03):365-374

15 Geiger AM, Nekhlyudov L, Herrinton LJ, et al. Quality of life after bilateral prophylactic mastectomy. Ann Surg Oncol 2007;14(02): 686-694

16 Tercyak KP, Peshkin BN, Brogan BM, et al. Quality of life after contralateral prophylactic mastectomy in newly diagnosed highrisk breast cancer patients who underwent BRCA1/2 gene testing. J Clin Oncol 2007;25(03):285-291

17 Isern AE, Tengrup I, Loman N, Olsson H, Ringberg A. Aesthetic outcome, patient satisfaction, and health-related quality of life in women at high risk undergoing prophylactic mastectomy and immediate breast reconstruction. J Plast Reconstr Aesthet Surg 2008;61(10):1177-1187

18 Frost MH, Hoskin TL, Hartmann LC, Degnim AC, Johnson JL, Boughey JC. Contralateral prophylactic mastectomy: long-term consistency of satisfaction and adverse effects and the significance of informed decision-making, quality of life, and personality traits. Ann Surg Oncol 2011;18(11):3110-3116

19 Wasteson E, Sandelin K, Brandberg Y, Wickman M, Arver B. High satisfaction rate ten years after bilateral prophylactic mastectomy a longitudinal study. Eur J Cancer Care (Engl) 2011;20(04):508-513

20 Spear SL, Schwarz KA, Venturi ML, Barbosa T, Al-Attar A. Prophylactic mastectomy and reconstruction: clinical outcomes and patient satisfaction. Plast Reconstr Surg 2008;122(01):1-9

21 Brandberg Y, Sandelin K, Erikson S, et al. Psychological reactions, quality of life, and body image after bilateral prophylactic mastectomy in women at high risk for breast cancer: a prospective 1year follow-up study. J Clin Oncol 2008;26(24):3943-3949

22 Gopie JP, Mureau MAM, Seynaeve C, et al. Body image issues after bilateral prophylactic mastectomy with breast reconstruction in healthy women at risk for hereditary breast cancer. Fam Cancer 2013;12(03):479-487

23 Soran A, Ibrahim A, Kanbour M, et al. Decision making and factors influencing long-term satisfaction with prophylactic mastectomy in women with breast cancer. Am J Clin Oncol 2015;38(02):179-183

24 Colakoglu S, Khansa I, Curtis MS, et al. Impact of complications on patient satisfaction in breast reconstruction. Plast Reconstr Surg 2011;127(04):1428-1436

25 Kwong A, Chu AT. What made her give up her breasts: a qualitative study on decisional considerations for contralateral prophylactic mastectomy among breast cancer survivors undergoing BRCA1/2 genetic testing. Asian Pac J Cancer Prev 2012;13(05):2241-2247

26 Nelson JA, Tchou J, Domchek S, Sonnad SS, Serletti JM, Wu LC. Breast reconstruction in bilateral prophylactic mastectomy patients: factors that influence decision making. J Plast Reconstr Aesthet Surg 2012;65(11):1481-1489

27 Koslow S, Pharmer LA, Scott AM, et al. Long-term patient-reported satisfaction after contralateral prophylactic mastectomy and implant reconstruction. Ann Surg Oncol 2013;20(11):3422-3429

28 Spurná Z, Dražan L, Foretová L, Dvorská L. [The effect of prophylactic mastectomy with recontruction on quality of life in BRCA positive women]. Klin Onkol 2012;25(Suppl):S74-S77

29 Unukovych D, Sandelin K, Liljegren A, et al. Contralateral prophylactic mastectomy in breast cancer patients with a family history: a prospective 2-years follow-up study of health related quality of life, sexuality and body image. Eur J Cancer 2012;48(17):3150-3156

30 Gahm J, Wickman M, Brandberg Y. Bilateral prophylactic mastectomy in women with inherited risk of breast cancer-prevalence of pain and discomfort, impact on sexuality, quality of life and feelings of regret two years after surgery. Breast 2010;19(06):462-469 
31 Montgomery LL, Tran KN, Heelan MC, et al. Issues of regret in women with contralateral prophylactic mastectomies. Ann Surg Oncol 1999;6(06):546-552

32 Pusic AL, Chen CM, Cano S, et al. Measuring quality of life in cosmetic and reconstructive breast surgery: a systematic review of patient-reported outcomes instruments. Plast Reconstr Surg 2007;120(04):823-837, discussion 838-839

33 Pusic AL, Klassen AF, Scott AM, Klok JA, Cordeiro PG, Cano SJ. Development of a new patient-reported outcome measure for breast surgery: the BREAST-Q. Plast Reconstr Surg 2009;124(02): 345-353

34 The Breast-Q. Available at: http://www.mskcc.org/mskcc/shared/ Breast-Q/scoreBQ.html. Accessed October 15, 2013

35 Garbay JR, Rietjens M, Petit JY. [Esthetic results of breast reconstruction after amputation for cancer. 323 cases]. J Gynecol Obstet Biol Reprod (Paris) 1992;21(04):405-412

36 Lostumbo L, Carbine NE, Wallace J. Prophylactic mastectomy for the prevention of breast cancer. Cochrane Database Syst Rev 2010;(11):CD002748

37 Sahin I, Isik S, Alhan D, Yıldız R, Aykan A, Ozturk E. One-staged silicone implant breast reconstruction following bilateral nipple- sparing prophylactic mastectomy in patients at high-risk for breast cancer. Aesthetic Plast Surg 2013;37(02):303-311

38 Pusic AL, Klassen AF, Snell L, et al. Measuring and managing patient expectations for breast reconstruction: impact on quality of life and patient satisfaction. Expert Rev Pharmacoecon Outcomes Res 2012;12(02):149-158

39 Salonen P, Tarkka MT, Kellokumpu-Lehtinen PL, Koivisto AM, Aalto P, Kaunonen M. Effect of social support on changes in quality of life in early breast cancer patients: a longitudinal study. Scand J Caring Sci 2013;27(02):396-405

40 Safarinejad MR, Shafiei N, Safarinejad S. Quality of life and sexual functioning in young women with early-stage breast cancer 1 year after lumpectomy. Psychooncology 2013;22(06): 1242-1248

41 Rosson GD, Shridharani SM, Magarakis M, et al. Quality of life before reconstructive breast surgery: a preoperative comparison of patients with immediate, delayed, and major revision reconstruction. Microsurgery 2013;33(04):253-258

42 Norman GR, Sloan JA, Wyrwich KW. Interpretation of changes in health-related quality of life: the remarkable universality of half a standard deviation. Med Care 2003;41(05):582-592 